ARTICLE

\title{
Rashba coupling amplification by a staggered crystal field
}

David Santos-Cottin ${ }^{1}$, Michele Casula1, Gabriel Lantz², Yannick Klein¹, Luca Petaccia ${ }^{3}$, Patrick Le Fèvre ${ }^{4}$, François Bertran ${ }^{4}$, Evangelos Papalazarou ${ }^{2}$, Marino Marsi ${ }^{2} \&$ Andrea Gauzzi $^{1}$

There has been increasing interest in materials where relativistic effects induce non-trivial electronic states with promise for spintronics applications. One example is the splitting of bands with opposite spin chirality produced by the Rashba spin-orbit coupling in asymmetric potentials. Sizable splittings have been hitherto obtained using either heavy elements, where this coupling is intrinsically strong, or large surface electric fields. Here by means of angular resolved photoemission spectroscopy and first-principles calculations, we give evidence of a large Rashba coupling of $0.25 \mathrm{eV} \AA$, leading to a remarkable band splitting up to $0.15 \mathrm{eV}$ with hidden spin-chiral polarization in centrosymmetric $\mathrm{BaNiS}_{2}$. This is explained by a huge staggered crystal field of $1.4 \mathrm{~V} \AA^{-1}$, produced by a gliding plane symmetry, that breaks inversion symmetry at the $\mathrm{Ni}$ site. This unexpected result in the absence of heavy elements demonstrates an effective mechanism of Rashba coupling amplification that may foster spin-orbit band engineering.

\footnotetext{
${ }^{1}$ IMPMC, Sorbonne Universités, Université Pierre et Marie Curie, CNRS, IRD, MNHN, 4 place Jussieu, 75252 Paris, France. ${ }^{2}$ Laboratoire de Physique des Solides, CNRS, Université Paris-Sud, Université Paris-Saclay, 91405 Orsay, France. ${ }^{3}$ Elettra Sincrotrone Trieste, Strada Statale 14 km $163.5,34149$ Trieste, Italy. ${ }^{4}$ Synchrotron SOLEIL, L'Orme des Merisiers, BP 48 Saint-Aubin, 91192 Gif-sur-Yvette Cedex, France. Correspondence and requests for materials should be addressed to D.S.-C. (email: David.Santos-Cottin@espci.fr) or to A.G. (email: andrea.gauzzi@upmc.fr).
} 
T he discovery of the integer quantum Hall effect in field-effect transistors ${ }^{1}$ initiated a novel approach to the electronic properties of crystalline solids based on the topological properties of Bloch states ${ }^{2}$, which fostered the discovery of further remarkable effects, such as the fractional quantum Hall effect ${ }^{3}$, the quantum spin Hall effect ${ }^{4}$ and charge antilocalization ${ }^{5,6}$. These effects suggest novel device concepts for quantum computation and spintronics, where information is carried without dissipation by using spin, instead of charge, currents $^{7}$. In this context, there has been an increasing interest in systems where the spin-orbit (SO) coupling leads to the opening of gaps at the Fermi surface and to spin-polarized bands ${ }^{8}$. An attractive possibility is the splitting of electronic states with opposite spin chirality $\chi=\hat{\boldsymbol{\sigma}} \cdot \hat{\mathbf{k}} \times \hat{\mathbf{z}}= \pm 1$ in nonmagnetic materials by the SO Rashba effect. The Rashba Hamiltonian, $\hat{\mathcal{H}}_{\mathrm{R}}=\alpha_{\mathrm{R}} \hat{\mathbf{z}} \cdot \hat{\boldsymbol{\sigma}} \times \hat{\mathbf{k}}=\beta \mathbf{E} \cdot \hat{\boldsymbol{\sigma}} \times \mathbf{k}$, where $\alpha_{\mathrm{R}}$ and $\beta$ are constants and $\hat{\boldsymbol{\sigma}}$ is the spin vector operator composed of the Pauli matrices $\hat{\sigma}_{x}$, $\hat{\sigma}_{y}$ and $\hat{\sigma}_{z}$, requires an electric field $\mathbf{E}=E_{z} \hat{\mathbf{z}}$ that breaks the inversion symmetry along the $z$-direction perpendicular to the plane containing the spin $\boldsymbol{\sigma}$ and the electron wave vector $\mathbf{k}$.

It follows that typical Rashba systems are either bulk noncentrosymmetric crystals or surfaces, referred to as bulk or surface inversion asymmetry systems, respectively ${ }^{8}$. Notable examples are the layered polar semiconductor $\mathrm{BiTeI}^{9}$ and the two-dimensional electron gas formed at the surface of $\mathrm{SrTiO}_{3}$ (ref. 10), where Rashba splittings as large as $\Delta \varepsilon \approx 100 \mathrm{meV}$ have been reported. These large splittings are due to the presence of heavy elements (and thus to large SO couplings) in the former case and to a large electric field $E_{z} \approx 10 \mathrm{mV} \AA^{-1}$ at the surface in the latter case. In fact, as noted recently by Zhang et al. ${ }^{11}$, SO coupling effects are governed by the local symmetry of the potential felt by the electron, rather than by the symmetry of the bulk crystal. To the best of our knowledge, the first experimental evidence of this circumstance has been given by Riley et al. ${ }^{12}$ who observed spin-polarized bands in the layered system $2 \mathrm{H}-\mathrm{WSe}_{2}$. This polarization, not expected in the centrosymmetric $P 6_{3} / \mathrm{mmc}$ structure of $2 \mathrm{H}-\mathrm{WSe}_{2}$, is explained by the confinement of the electron states within a single $\mathrm{WSe}_{2}$ layer with local inversion asymmetry (LIA) ${ }^{13}$.

In the present work, we consider the quasi-two-dimensional semi-metal $\mathrm{BaNiS}_{2}$ (ref. 14), precursor of the metal-insulator transition (MIT) observed in the $\mathrm{BaCo}_{1-x} \mathrm{Ni}_{x} \mathrm{~S}_{2}$ system on the partial substitution of $\mathrm{Ni}$ for Co at $x_{\mathrm{cr}}=0.22$ (ref. 15). This MIT has attracted a great deal of interest for the similarity of its temperature-doping $(T-x)$ phase diagram to that of cuprate ${ }^{16}$, pnictide $^{17}$ and heavy-fermion ${ }^{18}$ superconductors, though it remains little studied. To the best of our knowledge, the experimental band structure is not available for $\mathrm{BaNiS}_{2}$ and first-principles calculations have been hitherto limited to a simple density functional theory (DFT) approach in the local density approximation neglecting the Hubbard repulsion term, $U$, and relativistic SO effects ${ }^{19,20}$. The strong local Coulomb repulsion is expected to play a crucial role on the MIT, as indicated by dynamical mean field theory calculations on the insulating phase $\mathrm{BaCoS}_{2}$ (refs 21,22). To elucidate these open issues, the objective of the present study is to provide a first thorough description of the electronic structure of $\mathrm{BaNiS}_{2}$. Previous calculations show that one challenge is a complex multiband structure. On the other hand, a favourable condition of this study is the absence of disorder caused by chemical substitutions and a simple tetragonal structure. By means of angular-resolved photoemission spectroscopy (ARPES) on high-quality single crystals, we find a very large Rashba splitting up to $\Delta \varepsilon \approx 150 \mathrm{meV}$, which is unexpected in a centrosymmetric system without electronically active heavy elements. This observation is explained by a very effective LIA mechanism associated with a peculiar non-symmorphic square-pyramidal structure with a gliding plane symmetry. Unlike $\mathrm{WSe}_{2}$, in the present case, the large splittings observed are not due to the presence of heavy elements but to a huge staggered crystal field at the $\mathrm{Ni}$ site, $E_{z} \approx 1.4 \mathrm{eV} \AA^{-1}$, associated with the pyramidal coordination of the $\mathrm{Ni}$ ions. The mechanism unveiled by the present results, therefore, opens new possibilities to tune effectively the topological order of solids without the constraint of using either heavy elements or external fields.

\section{Results}

$\boldsymbol{A} \boldsymbol{b}$ initio calculations. Before presenting the ARPES results and the evidence of Rashba effect, we recall the salient features of the electronic structure of $\mathrm{BaNiS}_{2}$ that we recalculated in the generalized gradient approximation (GGA) of DFT supplemented by the Hubbard repulsion, $U$, and by the Hund coupling, $J$. In Fig. 1a-c, we show the crystal structure, the calculated Fermi surface, the band structure and the total and projected density of states (DOS) for each $d$-orbital of Ni. The parameters of the local interaction matrix $(U=3 \mathrm{eV}, J=0.95 \mathrm{eV})$ were estimated from absorption data ${ }^{23}$. In the band structure of Fig. 1c, false colours represent the $d$-orbital component of each band. We use a reference system for the $d$-orbitals where the $x(y)$ axis coincides with the $a(b)$ axis, while the $z$ axis coincides with the $c$ axis and points towards the apical sulphur atom. All $d$-orbitals, except the low-lying $x y$ one, contribute to the DOS at the Fermi level, $\varepsilon_{\mathrm{F}}$, and hybridize strongly with the $3 p$ orbitals of the sulphur atoms. In Fig. 1c, the relevant bands near $\varepsilon_{\mathrm{F}}$ are labelled in Schönflies notation for the irreducible representations (IR) of the little point group of the corresponding $\mathbf{k}$-vector ${ }^{24}$. We first note two degenerate hole-like bands with $\mathrm{E}_{\mathrm{g}}\left(d_{x z}\right.$ and $\left.d_{y z}\right)$ character at the $\mathrm{D}_{4 \mathrm{~h}}$ symmetry $\Gamma$ point and with $\mathrm{E}$ character along the $\Lambda$ direction of $\mathrm{C}_{4 \mathrm{v}}$ symmetry; along the $\Delta$ (or $U$ ) and $\Sigma$ (or $S$ ) directions of lower $C_{2 v}$ symmetry, these bands are split into $B_{1}$ and $\mathrm{B}_{2}$ bands. Second, at $\mathrm{X}$ (or R) two bands with $\mathrm{A}_{\mathrm{g}}$ character carrying $d_{x^{2}-y^{2}}$ and $d_{z^{2}}$ components split into two $\mathrm{A}_{1}$ bands along $\Delta$ (or $U$ ). Finally, along $\Sigma$ (or $S$ ), two $A_{1}$ electron-like bands carrying $d_{x^{2}-y^{2}}$ and $d_{z^{2}}$ components, respectively, display a linear dispersion and cross at a point denoted Q, located almost exactly at $\varepsilon_{\mathrm{F}}$ between the $\Gamma$ and $\mathrm{M}$ points (or between $\mathrm{Z}$ and $\mathrm{A}$ ).

The resulting Fermi surface consists of four sheets. The $E_{g}$ band forms a hole pocket centred at $\Gamma$ with a peculiar shape similar to that of a carambola fruit. As a result of the $k_{z}$ dispersion along $\Lambda$, this band forms an electron pocket centred at $Z$ with square-shaped section. The $\mathrm{A}_{g}$ bands form a strongly oblated electron pocket centred at R. Finally, the two linearly dispersing $\mathrm{A}_{1}$ bands with $d_{x^{2}-y^{2}}$ and $d_{z^{2}}$ component form a Dirac-like cone with elliptic-like section and hole character. The origin $\mathrm{Q}$ of the cone is located near $k_{z}=0$. All pockets are small, which leads to semimetallic properties, as apparent from the pronounced dip of the DOS at $\varepsilon_{\mathrm{F}}$, and to enhanced electronic correlations. Each band is at least twofold degenerate in the orbital sector since the glide reflection plane of the $\mathrm{P} 4 / \mathrm{nmm}$ symmetry generates two $\mathrm{Ni}$ positions, $\boldsymbol{r}_{1}=(1 / 4,1 / 4, z)$ and $\boldsymbol{r}_{2}=(3 / 4,3 / 4,-z)$, in the unit cell.

Angular resolved photoemission spectroscopy. To obtain high-quality spectra, we optimized the growth of $\mathrm{BaNiS}_{2}$ single crystals as described in the experimental methods section. We successfully measured ARPES spectra on several high-purity single crystals cleaved in situ at low temperature within the $a b$ plane, which allowed us to measure the in-plane band dispersion, $\varepsilon_{\mathbf{k}}$. As an indication of the improved purity of these crystals, resistivity measurements (see Methods section) yielded residual resistivity ratios $R R R \approx 12-17$ significantly larger than those $\sim 4$ reported previously ${ }^{14,25}$. In Fig. 2, we show the $26 \mathrm{eV}$ ARPES 
a

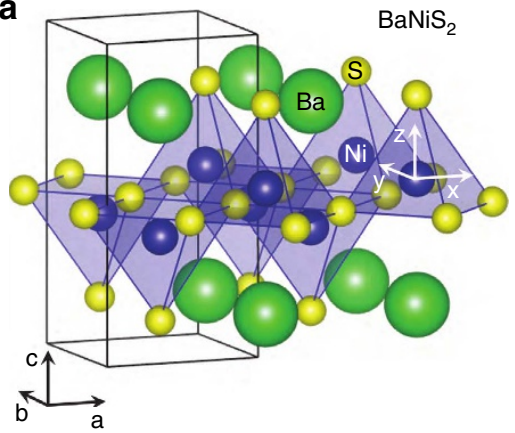

b

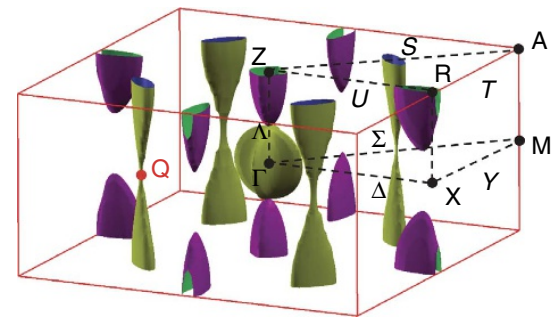

C

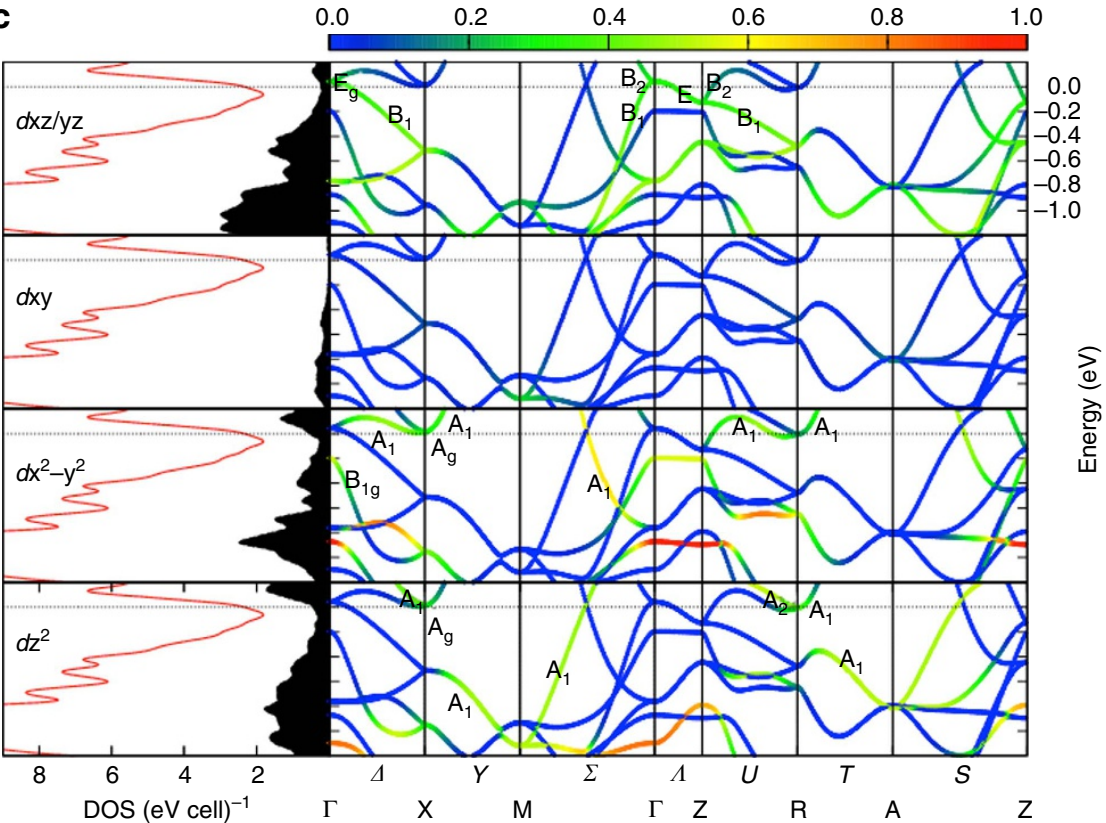

Figure 1 | Crystal and electronic structures of $\mathbf{B a N i S}_{2}$. (a) Tetragonal P4/nmm structure of $\mathrm{BaNiS}_{2}$. Note the staggered orientation of the NiS 5 pyramids. Details of the structure are given in ref. 14. (b) Fermi surface calculated in the GGA $+U$ approximation without spin-orbit coupling. Hole and electron pockets are indicated in yellow and purple, respectively. (c) Calculated electronic bands, total (red line) and projected (black area) density of states for each $3 d$ orbital. False colours indicate the $d$-orbital component for each band. The symmetry character of the relevant bands near the Fermi level, indicated by a dotted line, are labelled in Schönflies notation. Bold (italic) letters on the horizontal axis indicate high-symmetry points (lines).

bands measured in both $p$ - and $s$-polarizations along the $\Delta$ (or $U$ ) and $\Sigma$ (or $S$ ) symmetry directions. Consistent with the orbital character of the bands calculated without SO coupling (Fig. 1c), due to the different parity of the $d$-orbitals with respect to the scattering plane, the $d_{z^{2}}$ states are observed only in $p$-polarization along $\Sigma$, the $d_{x^{2}-y^{2}}$ state in $p$-polarization along $\Delta$ and in $s$-polarization along $\Sigma$ and the $d_{y z}$ and $d_{x z}$ states in both polarizations along $\Delta$.

A quantitative comparison between experimental and calculated bands is shown in Fig. 3a, where the previous GGA + $U$ bands are plotted together with the full ARPES band structure obtained by merging the $p$ - and $s$-polarized spectra of Fig. 2 . We noticed that the inclusion of the Hubbard repulsion $U$ term in the GGA calculations improves significantly the agreement with the experiment, especially concerning the energy position of the $B_{1 g}$ band at $\Gamma$ (or $Z$ ) and the dispersion of the $B_{1}$ band along $\Gamma-\mathrm{X}$ (or Z-R). To estimate the $k_{z}$ value, we calculated the band dispersion at different $k_{z}$ values, $k_{z}=0, \frac{1}{8}, \frac{1}{4}, \frac{3}{8}, \frac{1}{2}$ (in crystal units of $\frac{2 \pi}{c}$ ), and best agreement with the experiment is obtained for $k_{z}=\frac{3}{8}$. In Fig. 3a-c, the corresponding symmetry points and lines are labelled using primed letters.

As predicted by the calculations for $k_{z}=\frac{3}{8}$, the experimental Fermi surface of Fig. $3 \mathrm{~b}$ consists of the four hole pockets of the Dirac-like bands along $S$ and of the square-shaped electron pocket centred around Z'. A slight discrepancy between experiment and calculations concerns the hole pocket centred at R', not seen experimentally. According to the calculations, this may suggest that the $k_{z}$ value probed by the $26 \mathrm{eV}$ photons rather lies in the $0-\frac{1}{4} k_{z}$ range. In fact, in the calculated Fermi surface of Fig. 3d, both hole pockets at $\mathrm{Z}$ and at $\mathrm{R}$ shrink rapidly with decreasing $k_{z}$, so an accurate determination of the relative $k_{z}$ position of the bottom of these pockets goes beyond the capabilities of the present DFT method. In summary, the experimental data are explained by a pocket at $\mathrm{R}$ located at a higher $k_{z}$ value than calculated. This explanation is supported by the fact that the $100 \mathrm{ev}$ ARPES data do probe the bottom of the pocket, as seen in Fig. 4b. We conclude that the $26 \mathrm{eV}$ data of Figs $2-3$ and the $100 \mathrm{eV}$ data of Fig. $4 \mathrm{~b}$ probe the bands in the vicinity of $k_{z}=\frac{3}{8}$ and of $k_{z}=\frac{1}{2}$, respectively.

\section{Discussion}

We are now in the position of analysing one of the main features of the band structure, that is, the large $\approx 50 \mathrm{meV}$ splitting of the $E_{g}$ bands at $Z$, which suggests the importance of SO coupling 


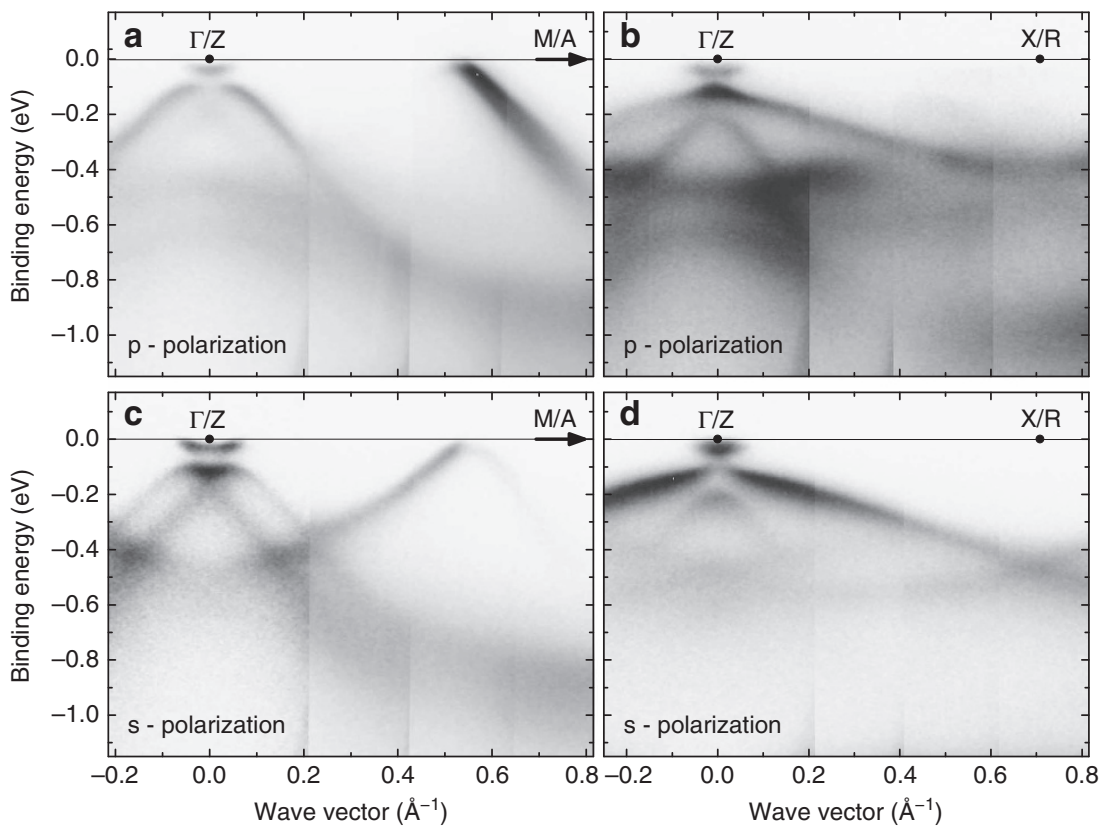

Figure 2 | ARPES bands of $\mathbf{B a N i S}_{\mathbf{2}}$. The band dispersion is shown along the $\Gamma \mathrm{M}(Z A)$ and $\Gamma X(Z R)$ symmetry directions obtained in both $p$ (a,b) and $s(\mathbf{c}, \mathbf{d})$ polarizations using a $26 \mathrm{eV}$ photon energy. Since the $k_{z}$ value probed is between 0 and $\pi / c$, symmetry points and symmetry directions referring to both values are indicated.
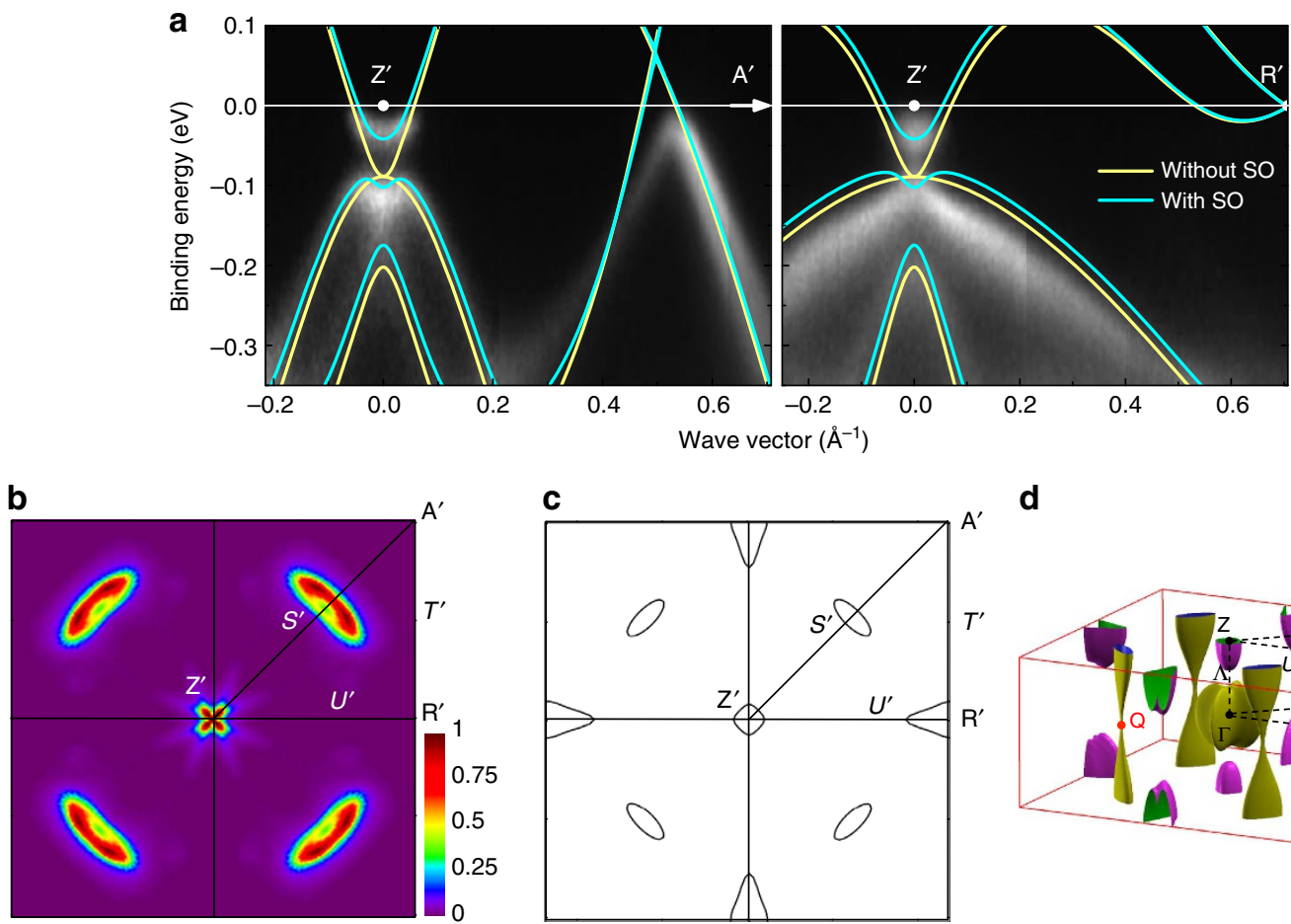

d

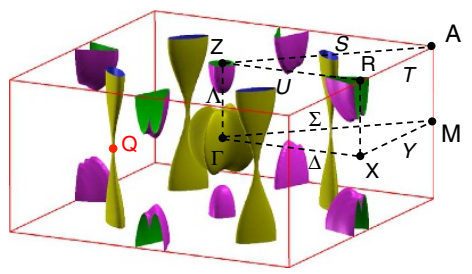

Figure 3 | Comparison with calculations. (a) $26 \mathrm{eV}$ ARPES bands compared with the GGA $+U$ bands along the $Z^{\prime}-A^{\prime}-R^{\prime}$ directions, corresponding to $k_{z}=\frac{3}{4} \frac{\pi}{c^{\prime}}$ calculated with (light blue line) and without (yellow line) spin-orbit coupling. The ARPES bands are obtained by merging the $s$ and $p$ polarized spectra of Fig. 2. The horizontal white line indicates $\varepsilon_{\mathrm{F}}$. Fermi surface measured experimentally (b) and calculated (c) at $k_{z}=\frac{3}{4} \frac{\pi}{c}$. (d) Full GGA $+U$ Fermi surface calculated with spin-orbit coupling. Hole and electron pockets are indicated in yellow and purple, respectively.

effects not included in the calculations. A straightforward symmetry analysis ${ }^{24,26}$ substantiates this hypothesis. As far as the SO band splittings is concerned, we consider the k-points with $k_{z}=\frac{1}{2}$, such as Z, on the same footing as the $\mathbf{k}^{\prime}$ points with $k_{z}=\frac{3}{8}$, such as Z'. The reason is that the predictions of splitting for the little groups $\mathcal{G}_{\mathbf{k}}$ of the former points are valid for the little groups $\mathcal{G}_{\mathbf{k}^{\prime}}$ of the latter points as well, as $\mathcal{G}_{\mathbf{k}^{\prime}}$ are subgroups of $\mathcal{G}_{\mathbf{k}}$. Thus, the symmetry analysis of the SO band splitting is the same for both $26 \mathrm{eV}$ and $100 \mathrm{eV}$ ARPES bands shown in Figs $3 \mathrm{a}$ and $4 \mathrm{~b}$, respectively. This analysis shows that, while the $\mathrm{E}_{\mathrm{g}}$ bands are degenerate in the non relativistic calculations, the product of their IR $Z_{5}$ of the $D_{4 h}$ little group for the $Z$ symmetry point with the 

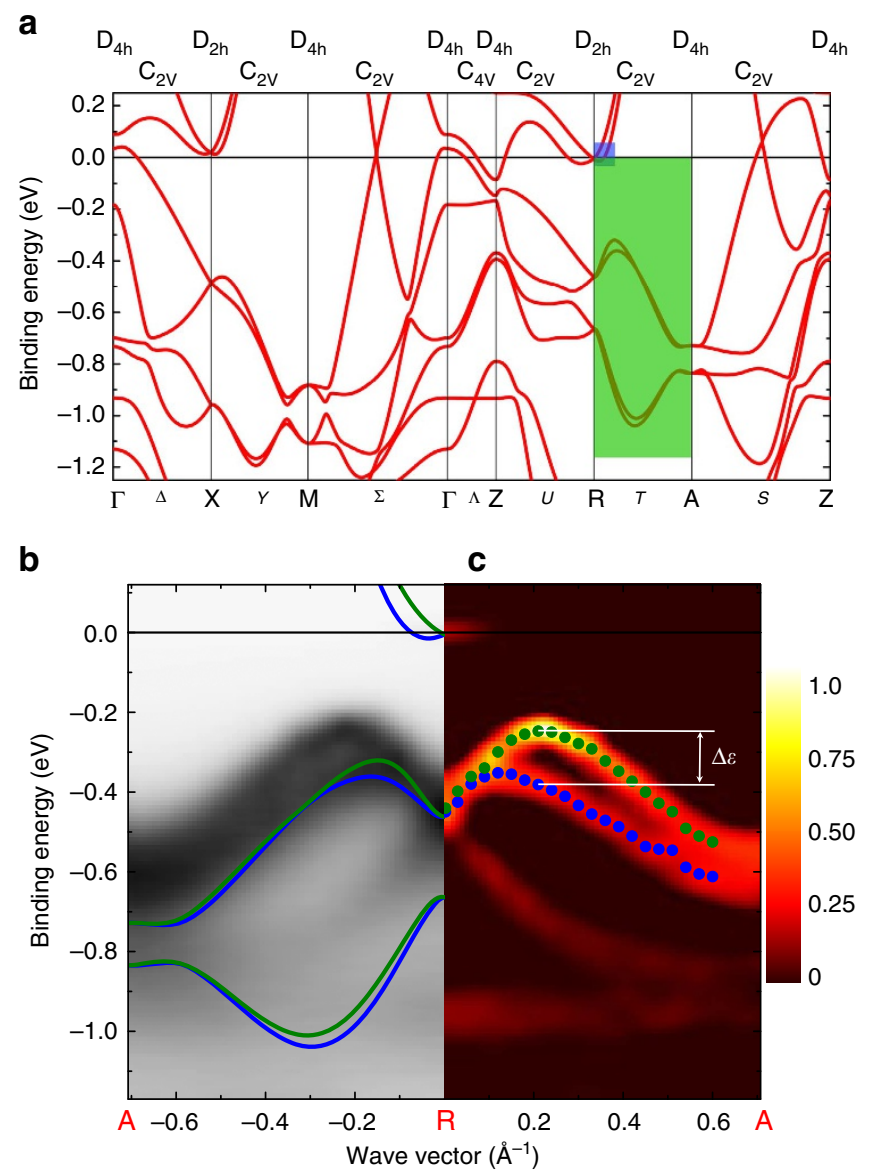

Figure 4 | Rashba-split bands. (a) GGA $+U$ band structure calculated with spin-orbit coupling along the main symmetry directions. The little groups of the symmetry points and lines are indicated on the top. The green and blue areas indicate the portions of the band structure shown in detail in Figs $4 \mathrm{~b}$ and $5 \mathrm{a}$, respectively. (b) Close-up of the calculated bands along the R-A symmetry direction (that is, for $k_{z}=\pi / c$ ) plotted together with the experimental ARPES bands measured using $100 \mathrm{eV}$ photons. Blue and green solid lines show the calculated Rashba-split band. (c) The experimental splitting of the hole-like band is put into evidence by plotting the second derivative of the energy distribution curves of the spectra of $\mathbf{b}$ at constant wave vector. Blue and green dots represent the peak position of the second derivative. The energy splitting, $\Delta \varepsilon \approx 150 \mathrm{meV}$, is indicated by white horizontal lines.

$D_{1 / 2}$ spin IR yields the following sum of IR's:

$$
Z_{5} \otimes D_{1 / 2}=Z_{11} \oplus Z_{12}
$$

We have then recalculated the band structure including the SO coupling. Remarkably, the result shown in Figs $3 \mathrm{a}$ and $4 \mathrm{a}$ is fully consistent with the above band splitting. The SO coupling also accounts for the experimental observation of a mixing of the $\mathrm{B}_{1 \mathrm{~g}}$ band below the Fermi level with the lower split $E_{g}$ band, clearly visible in the data at $Z$ (Fig. 3a). Indeed, by denoting $Z_{3}$ the IR of the $B_{1 \mathrm{~g}}$ band at $Z$, one obtains $Z_{3} \otimes D_{1 / 2}=Z_{12}$, so the two bands are described by the same $Z_{12}$ symmetry in the presence of $S O$ coupling.

The exciting feature of $\mathrm{BaNiS}_{2}$ revealed by the present work is that, in addition to the ordinary SO splittings discussed above, both the experimental bands and those calculated with SO coupling display Rashba splittings, as shown in Fig. 4a. We focus on the splitting of the electron-like band with $\mathrm{A}_{1}$ character with $d_{x^{2}-y^{2}}$ and $d_{z^{2}}$ components located just above $\varepsilon_{\mathrm{F}}$ near $\mathrm{R}$ along $T$ (or near X along $Y$ ) and of the hole-like band located in the same $k$ region $\approx 0.4 \mathrm{eV}$ below $\varepsilon_{\mathrm{F}}$. As seen in Fig. $1 \mathrm{c}$, near $\mathrm{R}$ the latter band displays $\mathrm{B}_{1}$ and $\mathrm{B}_{2}$ character with $d_{x z}$ and $d_{y z}$ components, respectively, that progressively evolves into $\mathrm{A}_{1}$ character with $d_{z^{2}}$ component moving to A. A close-up of the ARPES bands in Fig. $4 \mathrm{~b}$ shows that only the bottom of the electron-like band is observed, as mentioned above; so the prediction of Rashba effect cannot be verified experimentally for this band but a slight electron doping may be sufficient to probe it. On the other hand, the predicted Rashba splitting is clear in the hole-like band. The splittings in wave vector and in energy are as large as $\Delta k=0.2 \AA^{-1}$ and $\Delta \varepsilon \approx 150 \mathrm{meV}$, respectively. The latter value is even larger than in $\mathrm{BiTeI}^{9}$ or $\mathrm{SrTiO}_{3}$ (ref. 10), in spite of the absence of heavy elements or surfaces.

Both electron-like and hole-like bands are $k$ split along $\mathrm{R}-\mathrm{A}$ by the same Rashba interaction $\hat{\mathcal{H}}_{\mathrm{R}}=\alpha_{\mathrm{R}} \hat{\mathbf{z}} \cdot \hat{\boldsymbol{\sigma}} \times \mathbf{k}_{\mathrm{R}}$, where $\mathbf{k}_{\mathrm{R}}=\mathbf{k}-\mathbf{k}$ and $\mathbf{k}^{0}$ is the wave vector of the $\mathrm{R}$ point. While the electron-like upper band would be centred at $\mathrm{R}$ in the absence of SO correction, the hole-like band would have a maximum located between $\mathrm{R}$ and $\mathrm{A}$. As shown by the present calculations, this difference leads to two different shapes of the spinor band structure, illustrated in Figs $4 \mathrm{~b}$ and 5a, respectively. In Fig. 5a, the splitting of the electron-like band is decribed by the standard Mexican-hat shape. In Fig. 4b, the calculations predict a displaced split maximum for the hole-like band along the same direction, in agreement with the ARPES data. We fit the latter band using the band dispersion obtained by diagonalising the above Rashba Hamiltonian and find $\alpha_{\mathrm{R}}=0.26 \mathrm{eV} \AA$ from ARPES, in excellent agreement with the calculated value $0.28 \mathrm{eV} \AA$. The difference between experimental and calculated splitting is due to the limited accuracy of the GGA $+U$ approximation in calculating the unperturbed (that is, without SO) band dispersion and its mass renormalization. We computed the Rashba parameters also for the Mexican-hat splitting of the electron-like band, barely visible experimentally at $\varepsilon_{\mathrm{F}}$. For this band, we obtained $\alpha_{\mathrm{R}}=0.19$ $\mathrm{eV} \AA, k_{\mathrm{R}}=0.04 \AA^{-1}$ for the position of the minimum of the band with respect to $\mathrm{R}$, and $\varepsilon_{\mathrm{R}}=11 \mathrm{meV}$ for the depth of the Mexican hat (Fig. 5a).

Contrary to the usual Rashba case, here the spin degeneracy is not lifted, even for the electron-like Mexican-hat band, because every energy level is at least twofold degenerate due to the two-fold multiplicity of the $\mathrm{Ni}$ site. It follows that the Rashba Hamiltonian involves two-instead of one-spinors. Our calculations show (Fig. 5c) that the corresponding Bloch states, $\psi_{1, \mathbf{k}}$ and $\psi_{2, \mathbf{k}}$, are strongly localized either at $\boldsymbol{r}_{1}$ or at $\boldsymbol{r}_{2}$, where the dipolar crystal field (and the $\mathrm{NiS}_{5}$ pyramid) has opposite orientation, $\pm E_{z}$, along the $z$ direction. Owing to this localization, the Hamiltonian splits into two independent Rashba Hamiltonians, one for each position, with a staggered distribution of Rashba coupling $\alpha_{\mathrm{R}}$ forming a Rashba crystal in a square lattice. This situation corresponds to a LIA, as discussed by Zhang et al. $^{11}$ who called hidden the resulting compensated spin polarization to distinguish it from that of the usual bulk inversion asymmetry and surface inversion asymmetry cases.

In the present case, the spin chirality at the two Ni positions, $\boldsymbol{r}_{1}$ and $\boldsymbol{r}_{2}$, is opposite, so the net spin polarization of the unit cell is zero. For the Bloch state at $\boldsymbol{r}_{1}$, where $E_{z}>0$, the solution for the Rashba eigenspinor at a given wave vector $\mathbf{k}$ reads

$$
\psi_{1, \mathbf{k}}^{ \pm}=\frac{1}{\sqrt{2}}\left(\begin{array}{c} 
\pm i e^{-i \phi_{\mathbf{k}}} \\
1
\end{array}\right)
$$

where the superscript \pm denotes the upper and lower energy branch, respectively, and $\phi_{\mathbf{k}}=\arctan \frac{k_{y}-k_{y}^{0}}{k_{x}-k_{x}^{0}}$. Since $E_{z}$ has opposite sign at $\boldsymbol{r}_{2}$, it follows that $\psi_{1, \mathbf{k}}^{ \pm}=\psi_{2, \mathbf{k}}^{\mp}$; hence, each Rashba branch is made of two opposite spinors whose spatial part is assigned to one of the two Ni positions. This is illustrated in Fig. $5 c$, where 


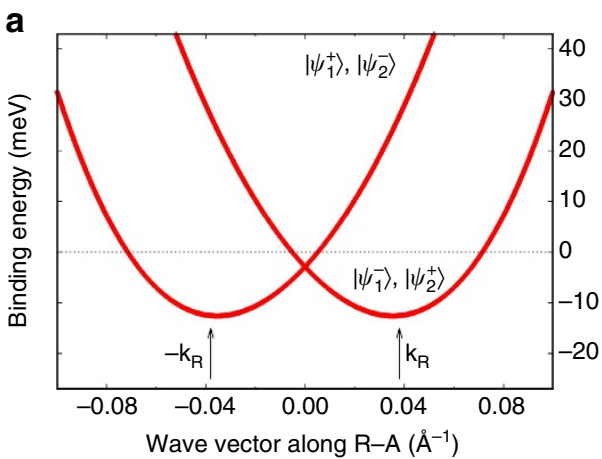

C

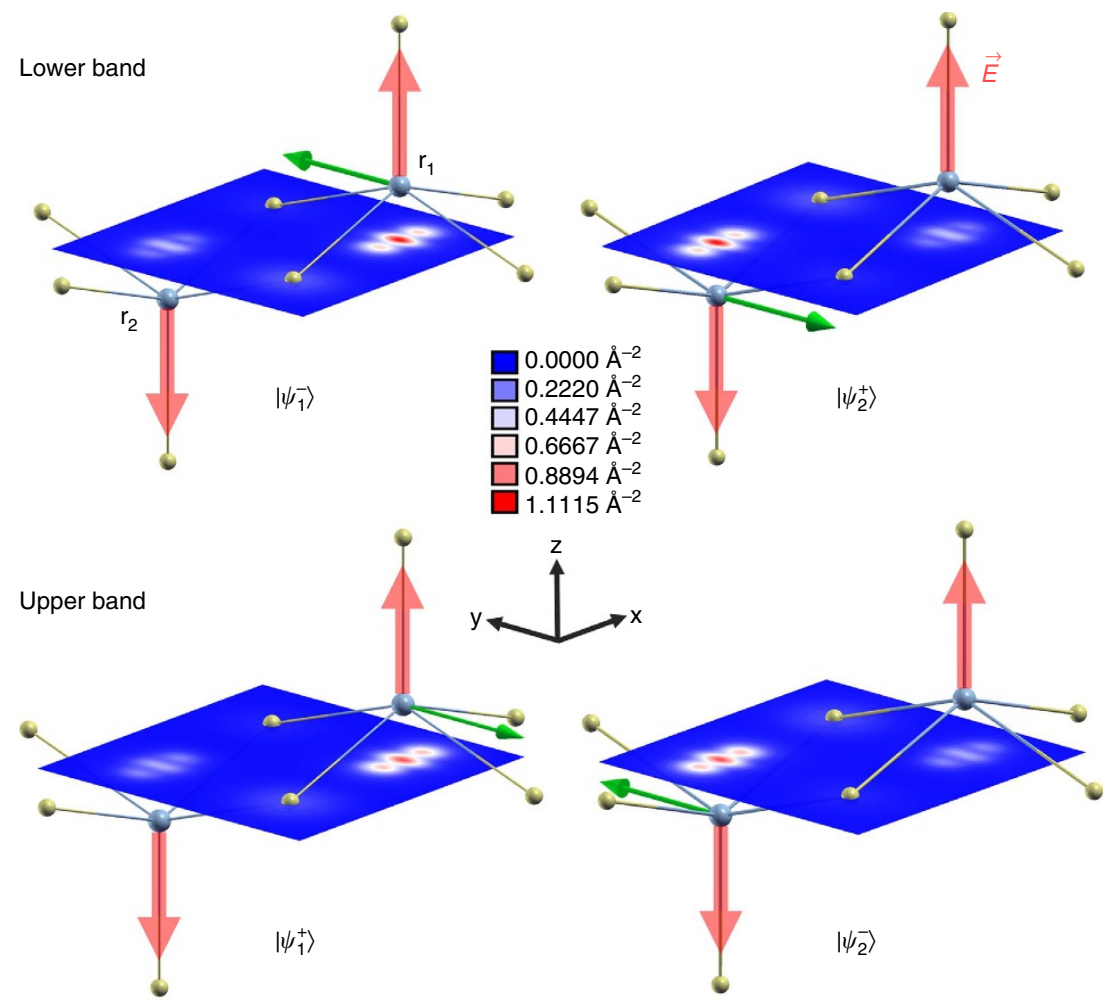

b 0.50

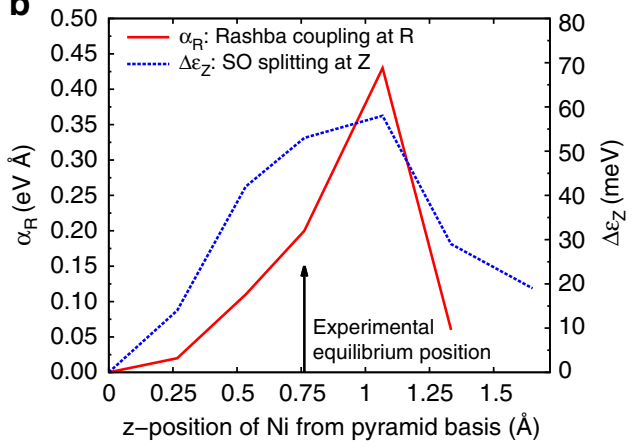


most stable structure corresponds to the largest Rashba splitting. This observation suggests an analogy with the Jahn-Teller systems where a gain of electronic energy drives a strain field that splits degenerate electronic states; here, the crystal field is the cause of the splitting.

Following Zhang et al. ${ }^{11}$, the present observation of Rashba effect in a centrosymmetric crystal arises from the LIA $\mathrm{C}_{4 \mathrm{v}}$ at the $\mathrm{Ni}$ site caused by the above staggered field, which contrasts the usual case of Rashba band splitting at $\Gamma$ in noncentrosymmetric crystals. The availability of a huge crystal field at the electronically active Ni site extends the possibilities of Rashba band engineering to light elements. The Rashba couplings obtained here could be further enhanced by substituting Ni for heavier $4 d$ or $5 d$ ions. To verify this expectation, we carried out preliminary GGA-DFT ab initio calculations on the hypothetical compounds $\mathrm{BaPdS}_{2}$ and $\mathrm{BaPtS}_{2}$ assuming that the crystal structure would be similar to that of $\mathrm{BaNiS}_{2}$. Our expectation is confirmed by a significant enhancement of the Rashba coupling for the electron-like band; namely, we obtain $\alpha_{\mathrm{R}}=0.35$ and $0.73 \mathrm{eV} \AA$ for the relaxed structure of the Pd and Pt compound, respectively. The corresponding band splittings are also dramatically enhanced up to $\Delta \varepsilon \approx 0.5 \mathrm{eV}$ in both compounds, which would enable a band structure engineering of solids in view of spintronics applications.

\section{Methods}

Single-crystal growth. High-purity $\mathrm{BaNiS}_{2}$ single crystals were synthesized by a self-flux method, as described elsewhere ${ }^{27,28}$. In brief, we first prepared a slightly off-stoichiometry mixture of finely grinded high-purity BaS (99.9\%), Ni (99.999\%) and $\mathrm{S}(99.9995 \%)$ powders in a graphite crucible. The chosen Ba:Ni:S = 0.10:0.425:0.475 composition ensures the crystal growth through the liquidus line of the pseudobinary phase diagram $\mathrm{BaNiS}_{2}-2 / 3 \mathrm{Ni}_{3} \mathrm{~S}_{2}+\delta$ system as shown in ref. 27. The crucible was then sealed under high vacuum at typical pressures

$P \sim 10^{-5} \mathrm{mbar}$ in a silica tube, heated to $1,000^{\circ} \mathrm{C}$ during 2 days and then quenched into water. The product was then grinded and pressed into a pellet for a second time by adding a $10 \%$ molar excess of sulphur and the aforementioned heat treatment was repeated. The silica tube was then slowly cooled from $1,000^{\circ} \mathrm{C}$ down to $800^{\circ} \mathrm{C}$ at a rate of $0.5-1{ }^{\circ} \mathrm{Ch}^{-1}$ and then quenched into water. The as-prepared melt contains platelet-like shiny black crystals of typical size $1 \times 1 \times 0.1 \mathrm{~mm}^{3}$ that can be mechanically removed from the melt. The crystalline quality, chemical composition and impurity concentration were checked by means of single-crystal $\mathrm{X}$-ray diffraction, energy dispersive $\mathrm{X}$-ray analysis and resistivity measurements in the van der Pauw configuration.

\section{Angular resolved photoemission spectroscopy. The ARPES experiment was carried out on crystals freshly cleaved in situ within the $a b$-plane at $100 \mathrm{~K}$ in ultra-high vacuum, at pressures of $10^{-11} \mathrm{mbar}$ or better, at the BaDElPh beamline of the Elettra synchrotron in Trieste, Italy, and at the Cassiopée beamline of the SOLEIL synchrotron in Saint-Aubin, France. In the former experiment, we used an incident photon energy $h v=26 \mathrm{eV}$ with a $\Delta \varepsilon=5 \mathrm{meV}$ photon energy resolution. To determine the parity of the bands, the experiment was performed in both $p$ and $s$ photon polarizations. In the former case, the incoming electric field, perpendicular to the photon momentum, lies within the scattering plane defined by the photon momentum and by the photoelectron momentum. In the latter case, the electric field is perpendicular to the scattering plane. In our geometry, the $p$ polarization forms an angle of $45^{\circ}$ with the $a b$-plane when at normal emission, while the $s$ polarization is parallel to this plane. In the experiment conducted at SOLEIL, the photon energy was $100 \mathrm{eV}$ and the photon energy resolution was $\Delta \varepsilon=10 \mathrm{meV}$.}

$\boldsymbol{A} \boldsymbol{b}$ initio calculations. The $a b$ initio calculations have been carried out in the GGA-DFT framework with the Perdew-Burke-Ernzerhof functional, implemented in the Quantum ESPRESSO package ${ }^{29}$. In the GGA $+U$ calculations, we used the rotationally invariant formalism ${ }^{30}$ with the Hubbard matrix correlating the $\mathrm{Ni}$ $3 d$ orbitals and fulfilling the atomic spherical symmetries. The Hubbard parameters are $U=3 \mathrm{eV}, J=0.95 \mathrm{eV}$, and the $F_{4} / F_{2}$ Slater integral ratio is taken equal to the atomic value. $\mathrm{Ni}, \mathrm{Ba}$ and $\mathrm{S}$ atoms are described by norm-conserving pseudopotentials. The Ni pseudopotential has 10 valence electrons $\left(4 s^{2} 3 d^{8}\right)$ and non linear core corrections. For $\mathrm{Ba}$, the semi-core states have been explicitely included in the calculations. The $\mathrm{S}$ pseudopotential is constructed with the $3 s^{2} 3 p^{4}$ in-valence configuration. In the GGA $+U$ calculations with SO coupling, the $\mathrm{Ni}$ pseudopotential is fully relativistic and a non-collinear spin (spinor) formalism is used in the DFT framework. The geometry of the cell and the internal coordinates are taken from the experimental values reported previously ${ }^{14}$. The plane-wave cutoff is $120 \mathrm{Ry}$ for the wave function and $500 \mathrm{Ry}$ for the charge. A $8 \times 8 \times 8$ electron-momentum grid and a Methfessel-Paxton smearing of 0.01 Ry are used in the electronic integration during the self-consistent loop. Further calculations with a $16 \times 16 \times 16$ electron-momentum grid and tetrahedra interpolation have been performed for a more accurate determination of the Fermi level starting from a previously converged $8 \times 8 \times 8$ self-consistent electron density. For band structure and Fermi surface calculations, we carried out a Wannier interpolation of the $a b$ initio band structure by means of the Wannier90 (ref. 31) program on a

$N_{w}=4 \times 4 \times 4$ electron-momentum mesh by including all $\mathrm{Ni} d$ and $\mathrm{S} p$ states in a $11 \mathrm{eV}$ energy window, which allows to obtain highly accurate interpolated bands.

\section{References}

1. Klitzing, K. V., Dorda, G. \& Pepper, M. New method for high-accuracy determination of the fine-structure constant based on quantized hall resistance. Phys. Rev. Lett. 45, 494-497 (1980).

2. Wen, X.-G. Topological orders and edge excitations in fractional quantum Hall states. Adv. Phys. 44, 405-473 (1995).

3. Stormer, H. L., Tsui, D. C. \& Gossard, A. C. The fractional quantum Hall effect. Rev. Mod. Phys. 71, S298-S305 (1999).

4. Kane, C. L. \& Mele, E. J. $Z_{2}$ topological order and the quantum spin hall effect. Phys. Rev. Lett. 95, 146802 (2005).

5. Fu, L. \& Kane, C. L. Topological insulators with inversion symmetry. Phys. Rev. $B$ 76, 045302 (2007).

6. Chen, J. et al. Gate-voltage control of chemical potential and weak antilocalization in $\mathrm{Bi}_{2} \mathrm{Se}_{3}$. Phys. Rev. Lett. 105, 176602 (2010).

7. Hsieh, D. et al. A topological Dirac insulator in a quantum spin Hall phase. Nature 452, 970-U5 (2008).

8. Winkler, R. Spin-orbit Coupling Effects in Two-Dimensional Electron and Hole Systems. Springer Tracts in Modern Physics, Vol. 191 (Springer Verlag, 2003)

9. Ishizaka, K. et al. Giant Rashba-type spin splitting in bulk BiTeI. Nat. Mater. 10, 521-526 (2011).

10. Santander-Syro, A. F. et al. Giant spin splitting of the two-dimensional electron gas at the surface of $\mathrm{SrTiO}_{3}$. Nat. Mater. 13, 1085-1090 (2014).

11. Zhang, X., Liu, Q., Luo, J.-W., Freeman, A. J. \& Zunger, A. Hidden spin polarization in inversion-symmetric bulk crystals. Nat. Phys. 10, 387-393 (2014).

12. Riley, J. M. et al. Direct observation of spin-polarized bulk bands in an inversion-symmetric semiconductor. Nat. Phys. 10, 835-839 (2014).

13. Schaibley, J. \& Xu, X. Spintronics: a lucky break. Nat. Phys. 10, 798-799 (2014).

14. Grey, I. E. \& Steinfink, H. Crystal structure and properties of barium nickel sulfide, a square-pyramidal nickel(II) compound. J. Am. Chem. Soc. 92, 5093-5095 (1970).

15. Martinson, L. S., Schweitzer, J. W. \& Baenziger, N. C. Metal-insulator transitions in $\mathrm{BaCo}_{1-x} \mathrm{Ni}_{x} \mathrm{~S}_{2-y}$. Phys. Rev. Lett. 71, 125-128 (1993).

16. Lee, P. A., Nagaosa, N. \& Wen, X.-G. Doping a Mott insulator: Physics of high-temperature superconductivity. Rev. Mod. Phys. 78, 17-85 (2006).

17. Johnston, D. C. The puzzle of high temperature superconductivity in layered iron pnictides and chalcogenides. Adv. Phys. 59, 803-1061 (2010).

18. Joynt, R. \& Taillefer, L. The superconducting phases of $\mathrm{UPt}_{3}$. Rev. Mod. Phys. 74, 235-294 (2002).

19. Mattheiss, L. Electronic structure of quasi-two-dimensional $\mathrm{BaNiS}_{2}$. Solid State Commun. 93, 879-883 (1995).

20. Hase, I., Shirakawa, N. \& Nishihara, Y. Electronic structures of $\mathrm{BaNiS}_{2}$ and $\mathrm{BaCoS}_{2}$. J. Phys. Soc. Jpn 64, 2533-2540 (1995).

21. Zainullina, V. M. \& Korotin, M. A. Ground state of $\mathrm{BaCoS}_{2}$ as a set of energy-degenerate orbital-ordered configurations of $\mathrm{Co}^{2+}$ ions. Phys. Solid State 53, 978-984 (2011)

22. Zainullina, V. M., Skorikov, N. A. \& Korotin, M. A. Description of the pressureinduced insulator-metal transition in $\mathrm{BaCoS}_{2}$ within the LDA + DMFT approach. Phys. Solid State 54, 1864-1869 (2012).

23. Krishnakumar, S. R., Saha-Dasgupta, T., Shanthi, N., Mahadevan, P. \& Sarma, D. D. Electronic structure of and covalency driven metal-insulator transition in $\mathrm{BaCo}_{1-x} \mathrm{Ni}_{x} \mathrm{~S}_{2}$. Phys. Rev. B 63, 045111 (2001).

24. Atkins, P., Child, M. \& Phillips, C. Tables for Group Theory (Oxford University Press, 1970).

25. Martinson, L. S., Schweitzer, J. W. \& Baenziger, N. C. Properties of the layered $\mathrm{BaCo}_{1-x} \mathrm{Ni}_{x} \mathrm{~S}_{2}$ alloy system. Phys. Rev. B 54, 11265-11270 (1996).

26. Miller, S. C. \& Love, W. F. Tables of Irreducible Representations of Space Groups and Co-Representations of Magnetic Space Groups (Pruett Press, 1967).

27. Shamoto, S., Tanaka, S., Ueda, E. \& Sato, M. Single crystal growth of $\mathrm{BaNiS}_{2}$ J. Cryst. Growth 154, 197-201 (1995).

28. Shamoto, S., Tanaka, S., Ueda, E. \& Sato, M. Single crystal growth of $\mathrm{BaCo}_{1-x} \mathrm{Ni}_{x} \mathrm{~S}_{2}$. Physica C 263, 550-553 (1996).

29. Giannozzi, P. et al. QUANTUM ESPRESSO: a modular and open-source software project for quantum simulations of materials. J. Phys. Condens. Matter 21, 395502 (2009).

30. Liechtenstein, A. I., Anisimov, V. I. \& Zaanen, J. Density-functional theory and strong interactions: Orbital ordering in Mott-Hubbard insulators. Phys. Rev. B 52, R5467-R5470 (1995) 
31. Mostofi, A. A. et al. wannier90: A tool for obtaining maximally-localized Wannier functions. Comp. Phys. Commun. 178, 685-699 (2008).

\section{Acknowledgements}

We gratefully acknowledge financial support provided by the University Pierre and Marie Curie under the 'Programme émergence', by the EU/FP7 under contract Go Fast (Grant No. 280555) and by 'Investissement d'Avenir Labex PALM' (ANR-10-LABX0039-PALM). The computational resources used for this work have been provided by the GENCI under Project No. 096493. We are grateful to B. Baptiste for the single-crystal $\mathrm{X}$-ray diffraction measurements.

\section{Author contributions}

D.S-C. made and characterized the single crystals, led the ARPES data analysis and contributed in the figure preparation. M.C. carried out the first-principles calculations and led the theoretical analysis. G.L. led the ARPES data analysis and contributed in the figure preparation. G.L. L.P., P.L.-F., G.L., F.B., E.P. and M.M. contributed to the ARPES experiments. Y.K. contributed to the growth and characterization of the single crystals.
M.M. led the ARPES experiment. A.G. and M.C. led the analysis, wrote the paper and contributed in the figure preparation. A.G. proposed the research project.

\section{Additional information}

Competing financial interests: The authors declare no competing financial interests.

Reprints and permission information is available online at http://npg.nature.com/ reprintsandpermissions/

How to cite this article: Santos-Cottin, D. et al. Rashba coupling amplification by a staggered crystal field. Nat. Commun. 7:11258 doi: 10.1038/ncomms11258 (2016).

\section{(c) (1)}

This work is licensed under a Creative Commons Attribution 4.0 International License. The images or other third party material in this article are included in the article's Creative Commons license, unless indicated otherwise in the credit line; if the material is not included under the Creative Commons license, users will need to obtain permission from the license holder to reproduce the material. To view a copy of this license, visit http://creativecommons.org/licenses/by/4.0/ 